\title{
Development of Assessment and Screening Tool to Assist with Prevention and Identification of Charcot Foot in Type 2 Diabetics
}

\author{
Louise Wade DNP RN ${ }^{1,2, *}$ \\ ${ }^{1}$ Department of Nursing, Walden University, Baltimore, Maryland \\ ${ }^{2}$ Department of Nursing, West Texas A\&M University, Canyon, Texas
}

Received: September 15, 2017; Accepted: October 10, 2017; Published: November 06, 2017

*Corresponding author: Louise Wade DNP RN, Department of Nursing, Walden University, Baltimore, Maryland; E-mail: lwade@wtamu.edu

\begin{abstract}
Introduction: The DNP project is intended to improve patient care with the use of an assessment and screening tool and algorithm to aid in early identification of Charcot foot in the type 2 diabetic patient populations. The project involves the identification of a practice problem and the completion of a project that will lay ground work for future scholarship. According to the American Association of College of Nurses (AACN) (2006), "doctoral education in nursing is designed to prepare nurses for the highest level of leadership in practice and scientific inquiry. The DNP is a degree designed specifically to prepare individuals for specialized nursing practice, and The Essentials of Doctoral Education for Advanced Nursing Practice articulates the competencies for all nurses practicing at this level Assessment is a key element in nursing practice and this project addressed a need involving early identification of Charcot foot and referral for type 2 diabetic patients suffering from peripheral neuropathy.
\end{abstract}

\section{Problem Statement}

Diabetic neuropathy is the most prevalent complication of type 2 diabetes mellitus and affects equal to $50 \%$ of all type 2 diabetics. Peripheral neuropathy, or peripheral nerve damage, causes significant issues such as non healing wounds, major infections, amputations, and Charcot neuropathic osteoarthropathy (CN), commonly referred to as Charcot foot, which involves the soft tissue and bones of the foot and ankle and thus leads to permanent deformities. This may transpire if the bones in the feet suffer fractures and the foot becomes misaligned. Although experiencing a fracture would be extremely painful to most people, this particular condition can be painless to the diabetic patient since nerves were damaged from diabetes prior to the fracture.

\section{Purpose Statement}

In type $1 \mathrm{DM}$, distal polyneuropathy typically becomes symptomatic after many years of chronic prolonged hyperglycemia, whereas in type 2, it may be apparent after only a few years of known poor glycemic control or even at diagnosis" Symptoms affect sensory, motor, and autonomic systems of the body. When neuropathy progresses to Charcot foot, it becomes a serious, potential limb-threatening complication and during the acute phase, is considered to be an inflammatory syndrome. Due to the rarity of this condition, diagnosis and treatment poses a critical issue for healthcare practitioners including nurse practitioners [23,37]. For this reason, the intent of the project was the development of an assessment and screening tool, with integration of ADA recommendations, for nurse practitioners to promote early detection and treatment of Charcot foot so as to avoid additional injury and possible loss of lower limb or foot.

The foot or feet may subsequently lose muscle support, eventually converting to deformity. Diagnosis can sometimes be difficult due to the potential of mimicking other conditions like cellulites or deep venous thrombosis, and because diagnosis of a Charcot fracture cannot be made definitively until bone changes occur. Therefore, the focused problem in the project was inconsistency of healthcare providers in the recognition and referral of patients with potential Charcot foot.

\section{Goals and Outcomes}

The purpose of this project was to develop an assessment and screening tool and integrate recommendations set forth by the American Diabetes Association in an effort to assist nurse practitioners in the assessment, diagnosis, and treatment of Charcot foot in the diabetic patient with peripheral neuropathy. In doing so, the goal was to improve consistency of healthcare providers in the detection and treatment of patients with or at risk of Charcot foot.

This section outlines the process by which an assessment tool was developed, along with implementation and evaluation. No data was collected nor were participants involved as the project involved the development of an assessment tool to further assist NPs in the early detection, identification, and treatment of type 2 diabetic patients at risk of Charcot foot.

The outcomes that were used to determine goal attainment for the project included an evaluation planning step at the end 
of this DNP Project. The following outcomes were suggested as possible starting points for evaluation planning:

Outcome 1: Healthcare providers will identify, assess, and treat patients with Charcot foot.

Outcome 2: Healthcare providers will refer patients with Charcot foot to appropriate specialty for follow up care.

The detection of patient risks by nurses, which is "the ability of nurses to accurately identify signals can lead to early interventions so that harm to patients is minimized or circumvented" [4]. Nurses and nurse practitioners are at the forefront of patient assessment, which is the first opportunity for detection and intervention of potentially life-threatening illness and injuries. They have a responsibility to patients to be skilled in their assessment abilities and intervene when necessary. Charcot foot, although complex and often difficult to diagnose, is a major complication of diabetes that requires immediate treatment after a detailed and skilled assessment by competent healthcare professionals.

The project design was a qualitative approach, which provided an opportunity for nurse practitioners to share their experiences and challenges when assessing the adult diabetic patient population. "Qualitative methods offer the opportunity to obtain an in-depth understanding of patient experiences and may elicit a deeper understanding of patient's perceptions and behaviors and the meanings they attach to their experiences" [22].

\section{Background}

The American Diabetes Association reports that 60-70\% of people with diabetes suffer from peripheral nerve damage, which can progress to Charcot foot and an estimated $0.5 \%$ of these patients will actually advance to Charcot. In the majority of cases, onset occurs following the age of 50 and after having been diagnosed with diabetes for 15 to 20 years [34].

Despite the fact that uncontrolled diabetes and loss of proprioception is the main contributing factor leading to Charcot, researchers now believe other predisposing elements may increase the risk such as widespread atherosclerosis, inflammation caused by minor injury, infection, ulceration, or any other disorder in which blood flow is impeded [21]. Discovering the underlying etiology is a crucial aspect in successful treatment. The incidence and prevalence of Charcot is not known exactly but is estimated to affect $0.8-8 \%$ of the diabetic population. This number increases to $10 \%$ when radiographic studies are used in diabetics with neuropathy. In addition, studies have shown men and women are equally affected and typically in their 5th and 6th decades of life and having had diabetes for at least 10 years or more [18].

Charcot is a devastating complication of diabetic peripheral neuropathy that may affect a person's physical appearance and their ability to work and has the potential of having an effect on their mental capabilities as well. Patients are often left with feelings of depression, guilt from financial strains, and isolation.
In addition, patients suffering from Charcot experience a high rate of depression and anxiety due to physical mobility restraints and chronic pain. Male patients are at an even greater threat of these complications as a resulting from an inability to work and provide for their families financially [9].

Finally, studies show that mortality rates of individuals with Charcot arthroplasty are significantly higher than those who have simple diabetic foot ulcerations as well as those with type 2 diabetes lacking foot complications at all. The comparable rates are $28.3,37.0$, and $18.8 \%$ [37].

\section{Significance for Future Practice, Research and Social Change}

The American Diabetes Association has developed recommendations for all healthcare practitioners to follow regarding the diagnosis, treatment, and surgical intervention of Charcot foot with the goal of rapid identification of signs and symptoms and appropriate treatment regimens as a means of preventing further complications. The guidelines address the areas of diagnostics, medical therapy, and surgical treatment of active Charcot neuropathy and stress the importance of early recognition and offloading and prevention of recurrence or new episodes of $\mathrm{CN}$ or other diabetic foot complications. [37].

Accurate assessment of the diabetic foot is a complex process requiring skill, experience, and knowledge of not only the disease but also signs and symptoms of potential complications. The loss of sensation due to peripheral nerve damage makes it difficult for providers to diagnose issues as well as unseen internal problematic issues such as destruction of bone tissue and cartilage as a result of uncontrolled hyperglycemia. It is critical that diabetic patients are adequately censored and made mindful of the possible complications that derive from this disease. Through lessening the percentage of amputations and enhancing quality of life by way of education and consistent monitoring, there will be a decrease in the amount of money spent on the longterm support of the patient with diabetes [25]. However, many clinicians lack experience in the area of Charcot foot assessment and often consider it as simply "a diabetic foot".

There is a limited amount of scientific literature in regard to treatment protocols and guidelines for management of Charcot foot and ankle deformities and may be in part due to the presence of each individual case of Charcot of the foot and ankle [46]. Whereas many patients pose with obvious deformities, there are a higher number of those who have, little, or vague complaints, which add to the difficulty of accurate diagnosing for the practitioner.

Due to the fact that type 2 diabetics are at risk for numerous multisystem complications, all healthcare personnel, including nurse practitioners, have a responsibility to patients to be knowledgeable and competent in advanced assessment skills in hopes of preventing further complications [37]. The Charcot foot in diabetes poses many clinical challenges in its diagnosis and management. Despite the time that has passed since the first publication on pedal osteoarthropathy in 1883, we have much 
to learn about the pathophysiology, and little evidence exists on treatments of this disorder" (p. 2123). Identifying this problem in its initial stages is critical to effective treatment. Patients should contact a podiatrist at the earliest onset of symptoms. Occasionally, diagnosis is problematic given this condition is capable of mimicking other major disorders such as cellulitis or deep venous thrombosis, and especially since diagnosis of a Charcot fracture is unable to be made definitively until bone changes occur. The initial indications of the Charcot foot are frequently mild in nature, but can become abundantly more pronounced with unperceived repetitive trauma. Charcot foot typically worsens slowly, with age; rapid progression is uncommon, and should motivate a rapid re-evaluation. Since undiagnosed Charcot can advance considerably to grim outcomes including infection, deformity, amputations, disability, loss of employment, financial and mental strains, and life-long devastating effects, it is crucial for practitioners to be knowledgeable and skilled in assessment and treatment methods.

\section{Implications for Practice}

Due to the rarity and often overlooked complication of peripheral neuropathy known as Charcot foot, diagnosis and treatment poses a critical issue for healthcare practitioners including nurse practitioners (Rogers et al, 2011). Therefore, an assessment and screening tool is needed to serve as a guide for nurse practitioners to assist in the early recognition and treatment and to prevent further injury and possible loss of limb. Nurses' position in prevention of foot ulcers and amputations is imperative by means of education, screening high-risk populations, and assessment and intervention. Foot care education is vital for all diabetic patients but more so for those posing an increased threat due to neuropathy. Nurses can encourage and teach patients how to perform daily foot exams as well as consequences of untreated wounds or delay in care. According to the World Health Organization, diabetes is becoming an epidemic in most countries; therefore, evidence demonstrates significant consequences lie on both healthcare providers as well as communities as a whole [1].

Diabetic foot complications are key contributors to soaring morbidity and mortality rates. Without obvious signs of inflammation such as warmth, erythema, or function deficit, it is a demanding challenge for healthcare providers to diagnose Charcot foot. "Foot complications in people with diabetes can be difficult to treat and conventional therapies often fail, leading to amputations; thus, prevention of this condition is of paramount importance [19]. Advanced practice nurses must be willing to accept continuous new evidence and tools that will improve patient outcomes as an integral part of their practice. Patients rely on the knowledge and skill of healthcare professionals to ensure their well-being and positive outcomes. Assessment is a key element of the nursing role for all patient populations but more so for those individuals suffering from major diseases such as diabetes or other causes of peripheral neuropathy. According to the American Diabetes Association, $60-70 \%$ of people with diabetes acquire peripheral nerve impairment that can expand to Charcot foot and roughly $0.5 \%$ of these patients progress to
Charcot. This data is especially relevant to nursing practice and advanced assessment skills.

\section{Implications for Social Change}

Diabetic neuropathy has the utmost widespread effect of type 2 diabetes with up to $50 \%$ of all diabetic patients affected. As per the American Diabetes Association [ADA], type 2 diabetes involves uncompromising systemic consequences, such as peripheral neuropathy, which has the potential of leading to devastating injuries [4]. Due to the loss of sensation, patients are often unaware of wounds or other abnormalities and therefore; delay seeking treatment. Additionally, due to the rarity of Charcot foot, healthcare providers often dismiss or overlook this serious complication and initiate other forms of treatment. Current statistics indicates diabetes will continue to affect the United States population for the foreseeable future and is by no means limited to the United States [17]. The complications associated with this disease will also continue to be prevalent among those affected and it is the responsibility of providers to seek resources to assist them with accurate and appropriate diagnoses and treatment options.

As with many other chronic health conditions, the social and mental aspects of type 2 diabetes can be devastating for patients, families, and care givers alike. Diabetic treatment regimens must be maintained on a daily basis, despite social pressures, economic status, or distracting life events [44]. While type 2 diabetes typically develops or manifests in middle adulthood, this may significantly influence motivation to seek treatment and may require greater efforts or willingness to change. Even in the early phase, subtle complications such as foot calluses may appear to be minor and unimportant for the diabetic patient, thus delay in seeking treatment. Other facets to consider are the costs of medical management of wound care, potential vascular interventions, infection control, wound closure, off-loading, and alternative and adjunctive therapies.

Socioeconomic issues begin with extensive healing. For patients who are unaware of an injury, which progresses to an ulceration, "the average cost of treatment ranges from \$3609 to $\$ 27,721$ " [39]. Regardless of whether a patient is in need of complex therapy over an extended period of time or is simply required to be in some form of offloading device during the acute phase of Charcot foot, the potential for financial strain is inevitable. Complications of diabetic foot conditions are typically debilitating to patients, families, and caregivers alike. Patients are often times unable to continue working and have to rely on others or governmental assistance programs to sustain their daily lives. Others who are permanently disabled are forced to file for long-term social security disability, which is an extremely long and drawn out process that may or may not be approved initially. For those who gain approval, the length of time for their first payment is typically six to seven months. Early detection is vital in advancement to further injury and reduces the incidence of long-term or permanent disability. 


\section{Definition of Terms}

The principal terms used throughout this project included type 2 diabetes, peripheral neuropathy, Charcot foot, podiatric, acute, inflammatory, deformity, amputation, and offloading.

Type 2 diabetes is the most common form of diabetes and is defined as a condition in which the body fails to utilize insulin properly, otherwise known as insulin resistance. Typically, the pancreas produces an excess of insulin to accommodate but, over time it is adequately produce the body's requirement of insulin to maintain blood glucose at normal levels (ada.org, n.d.). Peripheral neuropathy refers to the destruction or dysfunction of peripheral nerves, which are damaged by uncontrolled elevated blood glucose levels, traumatic injuries, infections, metabolic problems and exposure to toxins (mayoclinic.org, n.d.). CharcotMarie-Tooth (Charcot Foot or CMT) is named for three physicians who were first to describe it in 1886: Jean-Martin Charcot, Pierre Marie, and Howard Henry Tooth $[11,12]$. It is defined as a serious and potentially life-threatening complication associated with diabetes, which is characterized by various degrees of bone, joint, soft tissue, foot and often ankle involvement and is derived from underlying neuropathy, trauma, and perturbations of bone metabolism and involves inflammation during the acute phase [37]. Podiatric refers to the specialty of a podiatrist who is a doctor of podiatric medicine (DPM), also known as a podiatric physician or surgeon. Podiatrists diagnose and treat conditions of the foot, ankle, and related structures of the leg [45]. Acute is characterized by sharpness or severity, sudden onset, short course, or requiring short-term medical care (as for serious illness or traumatic injury). Inflammatory refers to having to do with the body's response to either invading foreign substances (such as viruses or bacteria) or to direct injury of body tissue. Deformity is defined as the quality or state of being deformed, disfigured, or misshapen. Amputation is the accidental or intentional removal of a limb or body part. Offloading refers to taking the load off or transfer from one place to another such as reduction of pressure. Removing pressure from one area of the foot to another; effective reduction in pressure.

\section{Evidence-Based Practice Model}

The problem identified within the adult type 2 diabetic populations, who are at risk of Charcot foot, and the need for an assessment tool for healthcare practitioners is an example of the Iowa model of evidence-based practice. The Iowa model begins with a trigger or identified problem, which may also be a knowledge-based problem and involves the development of a team of stakeholders and a practice change is developed, implemented, and evaluated [24].

The Iowa Model for evidence-based practice includes knowledge and problem triggers, which prompt providers to evaluate current practices as well as promoting research when evidence is lacking [36]. "The Iowa Model of Research in Practice infuses research into practice to improve the quality of care, and is an outgrowth of the Quality Assurance Model Using Research (QAMUR). Research utilization is seen as an organizational process. Planned change principles are used to integrate research and practice. The model integrates evidence-based healthcare acknowledges and uses a multidisciplinary team approach" [15].

The Iowa Model was followed in a before and after design and included 19 baccalaureate nurses working on an endocrinology unit in which the primary patient population consisted of diabetics with chronic leg ulcers [42]. The focus of the study was whether evidence based practice training courses could improve nursing skills. Results indicated trained nurses can prevent significant complications in diabetic patients including amputations and other adverse effects by means of early recognition and treatment interventions.

This model has served as a reference for the project since the primary goal is directed at improving patient health and outcomes by identifying a trigger such as misdiagnosed Charcot foot, then integrating a multidisciplinary team to design an improvement plan such as assessment tool development and review of ADA policy and practice guidelines. "In this model, knowledge- and problem-focused triggers lead staff members to question current nursing practice and whether patient care can be improved through the use of research findings"[40]. Putting evidence into practice can be a complex process but necessary for improvements in healthcare and patient outcomes. The IOWA Model has been a valuable resource in the project by providing a systematic process to identify and address an issue in diabetic health.

\section{Theory}

Change is brought about in healthcare through various driving forces. The concept of identifying a problem and using evidence-based practice to implement change is an example of following the nursing process, which is a systematic approach to patient care with the goal of improving patient care. Lippitt's theory of change is a model of nursing that mirrors the nursing process and follows the same four process elements including assessment, planning, implementation, and evaluation [27]. The project has identified the problem of assessing the diabetic patient at risk of Charcot foot as a problematic area for nurse practitioners due to its complexity and challenges in diagnosis. The plan was to develop an assessment and screening tool to aid in early detection in those patients at risk or who display clinical manifestations correlating to Charcot foot. The planning stage is designed to utilize the screening algorithm to determine the correct treatment or referral action for the patient and followed up at an appropriate time, which is the final evaluation stage.

\section{Literature Search Strategy}

The search for literature was conducted electronically and used the following databases: CINAHL, Medline, PubMed, EBSCO, and Walden University Library. Articles older than 10 years were not considered and the terms used for the search were: diabetes, type 2 diabetes, diabetes statistics, neuropathy, peripheral neuropathy, diabetic complications, Charcot, Charcot foot, Charcot-Marie-Tooth, podiatry, podiatric, orthopedic complications, foot deformities, diabetic assessment forms, foot assessment, offloading, and peripheral neuropathy assessment. 


\section{Literature Review}

"Charcot neuroarthropathy is an often overlooked complication in diabetic patients with peripheral neuropathy. A group of experts reported that $25 \%$ of patients referred to their facility who had Charcot neuroarthropathy had not received a correct diagnosis at the referring institution. The incorrect diagnoses included infection, gout, arthritis, fracture, venous insufficiency, and tumor" [8]. This article focused in detail on the devastating effects of misdiagnosed Charcot and emphasized the importance of accurate assessment by healthcare providers. The authors also discussed a case in which a 53 year-old male presented to the emergency department with a 3 day history of pain, redness, and swelling to the foot and ankle andwas misdiagnosed with cellulitis, admitted to the hospital for a course of antibiotics, discharged home with oral antibiotics, seen at his primary healthcare providers office 2-3 more times for follow up then finally referred to an orthopedic specialist where they were accurately diagnosed with Charcot foot in the acute phase. By this time, there was irreversible extensive damage to the foot.

From 1999-2008 of patients who underwent either a below or above the knee amputation, 60\% suffered from diabetic neuropathy and had some type of trauma, non-healing wound or other complication such as Charcot foot. Based on an exhaustive review and analysis of the study, the primary issue for patients at a heightened chance of foot and ankle problems was the identification and referral to the appropriate specialist [33]. Healthcare professionals, including nurse practitioners, were among those who did not recognize potential issues, which delayed care and led to amputations of the 3,445 patients included in the study.

Symptoms of Charcot foot affect sensory, motor, and autonomic systems of the body. When neuropathy progresses to Charcot Arthropathy, it becomes a serious, potential limb-threatening complication and during the acute phase, is considered to be an inflammatory syndrome. Due to the rarity of this condition, diagnosis and treatment poses a critical issue for healthcare practitioners including nurse practitioners $[10,37]$. Therefore, an assessment and screening tool for nurse practitioners is needed to assist in the early recognition and treatment of Charcot foot to prevent further complications and possible loss of foot or lower extremity.

A case of Charcot in the Canadian Journal of Medicine in which a 59 year-old male reported complaints of a plantar ulcer for two months but after examination, his healthcare provider discovered that his foot was also deformed; however, the patient had such severe neuropathy that he felt no pain at all [30]. They go on to state early detection is essential and "prevention of disease progression remains the mainstay of treatment, including prompt immobilization, absolute non-weight bearing and professional foot care on a regular basis" (p. 1392). While even the slightest of infection, injury, or minor surgery may trigger the body's inflammatory response, without the protective barrier of pain being present, diabetic patients with sensory impairment are at greater risk of further injury and early recognition is crucial [21].
Another valid argument derives from a literature review which discussed suggestions to assist healthcare providers in making early diagnoses of Charcot foot, choosing the appropriate treatment regimen and reducing the incidence of further complications including amputations, sepsis and death [26]. "Charcot neuroarthropathy $(\mathrm{CN})$ continues to be a persistent challenge for clinicians, especially in its acute phase. The report indicated that the diagnosis of $\mathrm{CN}$ is missed in as many as $79 \%$ of cases and an accurate diagnosis can be delayed up to 29 weeks" (p. 9).

Accurate diagnosis of Charcot can often be challenging [18]. The authors stress the significance of patient and physician awareness in order to gain prompt diagnosis and lessen the burden of foot complications. "Charcot arthro neuropathy is a potentially limb-threatening condition which, beyond the emotional and social burden of physical dysfunction, has been associated with increased mortality" [18]. In addition, the article contains six practical point recommendations for clinicians to aid them in early detection and management and include: Charcot should be considered in every diabetic patient with neuropathy; irrespective of whether the diagnosis is only suspected, immediate offloading should be initiated; if plain $\mathrm{x}$-rays are negative, this should not deter offoading; education to patients and physicians to increase early detection will be beneficial; ulceration or infection in the plantar aspect of the foot should be avoided and; surgical intervention may be required (consult a podiatric specialist). A detailed foot assessment and documentation utilizing a specified assessment tool, which follows ADA guidelines by a skilled practitioner, is recommended for all diabetic patients.

Finally, many diabetic patients with existing neuropathy may present with other distracting issues such as foot ulcerations, swollen extremities, or have no complaints of pain or discomfort at all; clinicians still have the responsibility to perform a thorough examination of the diabetic foot and must be skilled in their assessment techniques [20]. Most complications of Charcot can be avoided with immediate treatment in the acute phase. While it is equally important to exclude other infectious processes or conditions such as DVT, "the overriding goal of treatment is to avoid amputation and prevent further deformity. Good outcomes can be managed with footwear that allows adequate gait and activity, thus sustaining overall quality of life" [20].

\section{Methods Approach: Introduction}

The purpose of this quality improvement project was to develop an assessment and screening tool and integrate ADA recommendations to assist healthcare providers in the early detection of Charcot foot for the diabetic population. The target population for this project was the adult diabetic population who have been diagnosed as having type 2 diabetes for at least ten years, being treated with either oral hypoglycemic or insulin therapy, and those either having or at risk of peripheral neuropathy. Inclusion also involved those patients with a history of or currently being treated for any type of foot ulceration, wound, and injury, complaints of foot or ankle pain, and patients 
who have a documented change of foot appearance. Stakeholders for this project were the patients, private insurance carriers, Medicare and Medicaid, podiatrists, and healthcare practitioners who provide care to diabetic patients. This section will outline how the project achieved these development activities.

The steps in the course of this project were as follows:

1.Assemble an interdisciplinary project team community of stakeholders to guide the project

2.Review of best practices of diabetic foot assessment as presented in evidence-based literature.

3.Integrate ADA policies and practice guidelines for the assessment, treatment, and referral of the diabetic patient with or at risk for developing, Charcot foot in conjunction with the project team

4.Develop an assessment tool of the diabetic foot in conjunction with the project team.

5.Develop an implementation plan in collaboration with the project team

6.Develop an evaluation plan in collaboration with the project team.

\section{Interdisciplinary Project Team}

The multidisciplinary team members, consisting of five members, who were invited to participate in the project based on their knowledge and expertise in the area of diabetes and management of complications. Disciplines included diabetes education, nurse practitioner, podiatry, IT computer personnel, and nursing informatics. Members were selected for their knowledge and experience in treating diabetic patients, medical specialty in podiatric medicine, computer technology, and informatics. Each member reviewed ADA treatment recommendations regarding early detection and intervention of patients at risk of Charcot foot as well as appropriate treatment regimens. Utilization of valid resources such as medical specialists and those having experience in one particular area is beneficial for ensuring all essential elements are included in the plan design. Interdisciplinary teamwork is "a dynamic process involving two or more health professionals with complementary backgrounds and skills, sharing common health goals and exercising concerted physical and mental effort in assessing, planning, or evaluating patient care" [31].

\section{Team Literature Review}

The interdisciplinary team members each received a copy of the goals and objectives for the project prior to development of an assessment tool and were led through the review of scholarly literature. Furthermore, a Gannt chart was dispersed to each member to illustrate the incidence of Charcot foot along with information from the American Diabetes Association and the American Podiatric Medical Association. All of the above was provided during an initial meeting with the interdisciplinary project team.

\section{Products}

Throughout the development of this project, I collaborated with a diverse group of experts who embodied the interdisciplinary team. The team members continuously provided input on the needs of the nurse practitioner stakeholders as well as feedback on the assessment and screening tool. The project was accepted and adopted by various NP clinicians in the geographical area who were willing to integrate it into their examination and treatment of adult type 2 diabetic patients. Furthermore, for those NPs who were utilizing electronic medical record systems, the project tools were incorporated as part of the patient assessment process for each visit.

\section{Appendix A:}

Appendix A. is an example of the computation of a Scale Content Validation Instrument (S-CVI) for a 10-Item scale with two expert raters, which was used by two expert raters for ranking and validation of this project (Table 1).

\section{Table 1:}

Appendix A: Computation of an S-CVI for a 10-Item Scale with Two Expert Raters

\begin{tabular}{|l|c|c|c|}
\hline & $\begin{array}{c}\text { Expert Rater } \\
\text { No 1 }\end{array}$ & $\begin{array}{c}\text { Expert Rater } \\
\text { No 2 }\end{array}$ & Total \\
\hline $\begin{array}{c}\text { Items rated 1 } \\
\text { or 2 }\end{array}$ & 2 & 0 & 2 \\
\hline $\begin{array}{l}\text { Items rated 3 } \\
\text { or 4 }\end{array}$ & 0 & 8 & 8 \\
\hline \multicolumn{1}{|c|}{ Total } & 2 & 8 & 10 \\
\hline $\begin{array}{l}\text { S-CVI 8/10 }=.80 \\
\text { S-CVI, content validity index for the scale. } \\
\text { Ratings of } 1=\text { not relevant } \\
\text { Ratings of } 2=\text { somewhat relevant } \\
\text { Ratings of } 3=\text { quite relevant } \\
\text { Ratings of } 4=\text { highly relevant }\end{array}$ & \\
\hline
\end{tabular}

\section{Appendix B:}

Appendix B. is the Charcot Foot Assessment and Screening Tool to be used by nurse practitioners in the adult type 2 diabetic patient population who are at risk due to neuro sensory deficits or other identified risk factors. The assessment and screening tool is based on the American Diabetes Associations' recommendations, which could be utilized in the clinical setting and integrated into the patient's electronic medical record [4]. Focus points on the assessment were derived from key features of the foot, which included monofilament points, evidence of outward physical abnormalities, and severity of peripheral neuropathy. Patients who cannot reliably detect application of the 5.07, 10-g monofilament to designated sites on the plantar surface of their feet are considered to have lost protective sensation" [29]. The assessment and screening tool also includes a recommendation for annual follow up or podiatric referral based on findings of the examination by way of a screening algorithm (Table 2) (Figure 1). 
Table 2: Appendix B: Charcot Foot Assessment and Screening Tool

\begin{tabular}{|c|c|}
\hline Patient__ & Management \\
\hline \multicolumn{2}{|l|}{ DOB _ Age } \\
\hline Diabetes Type & Insulin \\
\hline $\mathrm{PCP}$ & Diet__ \\
\hline \multicolumn{2}{|l|}{ Latest $\mathrm{HgA1C}$} \\
\hline Height___ Weight__ & \\
\hline \multicolumn{2}{|l|}{ SKIN } \\
\hline \multicolumn{2}{|l|}{ Turgor } \\
\hline \multicolumn{2}{|l|}{ Temperature } \\
\hline \multicolumn{2}{|l|}{ Calluses_____other } \\
\hline \multicolumn{2}{|l|}{ SENSORY } \\
\hline RIGHT FOOT & LEFT FOOT \\
\hline Sensation: Present ___ Absent & Sensation: Present___ Absent \\
\hline 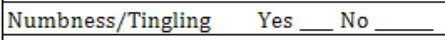 & Numbness/Tingling Yes _ No \\
\hline $\begin{array}{lll}\text { Burning } & \text { Yes_No_ No Burning }\end{array}$ & Yes_ No \\
\hline \multirow{2}{*}{\begin{tabular}{|ll} 
Sharp Pain & Yes_ No No \\
VASCUUAR &
\end{tabular}} & Sharp Pain $\quad$ Yes_ No N \\
\hline & VASCULAR \\
\hline RIGHT FOOT & LEFT FOOT \\
\hline Pedal pulse: Present ___ Absent & Pedal Pulse: Present __ Absent __ \\
\hline Edema: None__ $1+\ldots 2+\ldots 3+\ldots 4+\ldots$ & Edema: None_1 $1+{ }^{2+}{ }^{3+} 3+4+$ \\
\hline \multicolumn{2}{|l|}{ WOUNDS } \\
\hline RIGHT FOOT & LEFT FOOT \\
\hline Ulcer Yes _No_ & Ulcer Yes_ No \\
\hline Description (approx. size in mm) & Description (approx. size in mm) \\
\hline \multicolumn{2}{|l|}{ DEFORMITIES } \\
\hline RIGHT FOOT & LEFT FOOT \\
\hline Bunion Yes__ No & Yes__ No _ \\
\hline Corns $\quad$ Yes_ No & Corns \\
\hline \multirow{3}{*}{\begin{tabular}{|ll} 
Arch intact & Yes_ No \\
Other & \\
RISK LFVEI &
\end{tabular}} & Yes_No_ \\
\hline & Other \\
\hline & RISK LEVEL \\
\hline Low Risk & \\
\hline No sensory loss, ulcerations, or deformities & \\
\hline Treatment: Annual Assessment & \\
\hline Moderate Risk & \\
\hline Altered sensory, minimal structural deform & or beginning onset of ulcerations \\
\hline Treatment: Refer to Podiatry & \\
\hline High Risk & \\
\hline Impaired sensory, + numbness/tingling, he & d or active ulcerations, amputation, deformities \\
\hline Treatment: Refer to Podiatry & \\
\hline REFERRAL & \\
\hline Name of Podiatrist & Date Contacted \\
\hline Person making referral & Appointment Date \\
\hline $\begin{array}{l}\text { Special instructions or treatment given by } \\
\text { podiatrist }\end{array}$ & \\
\hline Signature of Provider & Date \\
\hline
\end{tabular}

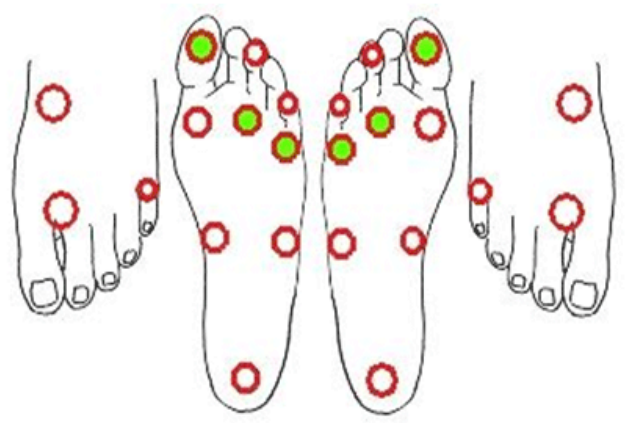

Figure 1:Neuropathy Monofilament Testing Sites

Monofilament testing for diabetic neuropathy using preferred testing locations colored green

If all sites are tested and the client feels the monofilament in each of the areas; then the score is $10 / 10$

If the monofilament is not felt in an area on the foot, this indicates loss of protective sensation (LOPS) in that area and requires referral to a podiatrist

Citation:Wade L (2017) Development of Assessment and Screening Tool to Assist with Prevention and Identification of Charcot Foot Page 7 of 11 in Type 2 Diabetics. J Endocrinol Diab 4(4): 1-11. 


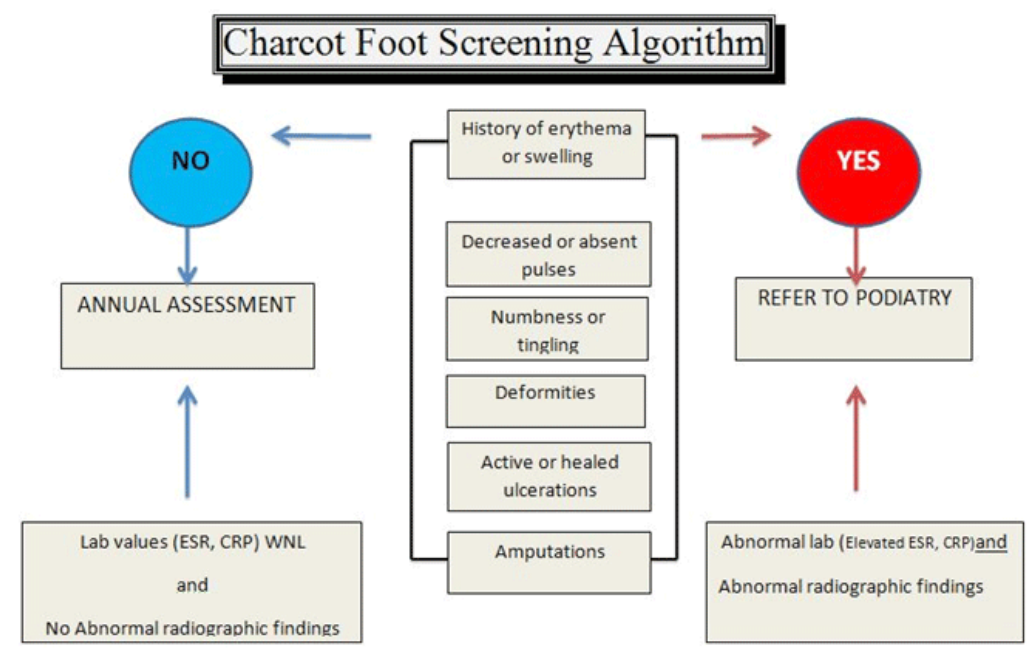

Figure 2: Appendix C: Charcot Foot Screening Algorithm

\section{Appendix C:}

Appendix C. is the Charcot Foot Screening Algorithm, which provides a clear guidance of treatment recommendations based on assessment findings. The algorithm is designed to guide practitioners in the care and treatment of patients based on assessment findings (Figure 2).

\section{Appendix D:}

Appendix D. includes Charcot Foot Assessment and Treatment Practice Guidelines. Due to the potential life threatening complications of type 2 diabetes and the complexity in diagnosing Charcot foot, it is imperative that healthcare professionals, such as nurse practitioners, be educated and competent in their assessment skills. According to the U. S. Department of Health and Human Services Health Resources and Services Administration Bureau of Health Professions Division of Nursing, "the adult nurse practitioner employs evidencebased clinical practice guidelines to guide screening activities, identifies health promotion needs, and provides anticipatory guidance and counseling addressing environmental, lifestyle, and developmental issues"[32]. Providers must maintain a level of expertise to aid in the diagnosis and treatment of all major disease complications (Figure 3).

\section{Appendix E:}

Appendix E. includes the implementation and evaluation plan for the project. Implementation is an essential component in the success of the scholarly project and addresses the key objectives for the project and makes for a smoother transition for completion of the project [28]. Equally important is the evaluation of the project by stakeholders to ensure the objectives are being met as well as opportunity for revisions. The intent of both the implementation and evaluation of the project is to make certain that evidence-based practices are being followed [16] (Table 3).
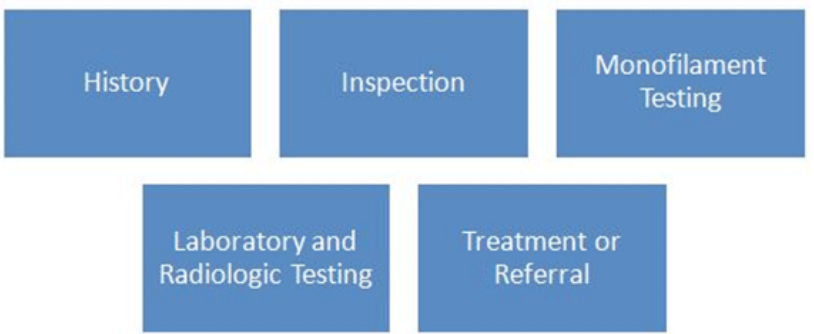

Figure 3: Appendix D: Charcot Foot Assessment and Treatment Practice Guidelines

HISTORY

-Edema or erythema

-Impaired neurovascular symptoms

-Recent injury or trauma

-Previous foot ulceration or amputation

INSPECTION

-Foot deformities or ulcerations

-Erythema or blisters

-Evidence of nonhealing areas

-Dryness, cracking, calluses, or fungal infections

\section{MONOFILAMENT TESTING}

-Recommended of four sites (1st, 3rd, and 5th metatarsal heads and plantar surface of distal hallux) be tested on each foot

-Apply the monofilament along the perimeter of (not on) the ulcer site

-Apply the monofilament to each site three times, including at least one additional

mock application in which no filament is applied

LABORATORY AND RADIOLOGIC TESTING

-ESR and CRP (Erythrocyte Sedimentation Rate and C-Reactive Protein)

-Radiologic exams on affected foot and ankle

\section{TREATMENT OR REFERRAL}

-Offloading of affected foot (orthopedic boot)

-Non weightbearing of affected foot (crutches)

-Referral to podiatry if identified as at risk or abnormal findings

-Annual foot examinations of no abnormal findings or risk factors identified 


\section{Development of Products}

The proposed assessment and screening tool, treatment algorithm, and treatment and practice guidelines were developed by the project team for later implementation with the nurse practitioner community in Northern Texas where there are currently 65 members of the Texas Panhandle Nurse Practitioners Association (TXPNPA). The assessment tool was distributed only to practitioners who are the primary care giver of adult type 2 diabetic patients. Part of the implementation phase included written forms of the assessment tool as well as the computerized version for those providers who have converted to electronic documentation. One benefit of utilizing the electronic medical record is the assessment tool will be a mandatory inclusion for providers, which will serve as an assessment reminder and hopefully reduce the number of undiagnosed cases. The computerized health information system being utilized at the health clinic has the capability of revisions to assessment templates and will be maintained by the clinic's computer personnel. These plans were presented to the project team for consideration and refinement. The final DNP Project included an implementation plan developed by the project team.

\section{Validation of Products}

Content validation in the area of Charcot foot is essential in order to go forth with the development of treatment and practice guidelines. One method of validation is the computation of a Scale Content Validation Instrument (S-CVI) for a 10-Item scale with two expert raters, which is defined as the proportion of items given a rating of quite/very relevant by both raters involved [35]. This particular method allows the entire scale of items, up to 10 , to be ranked by the raters as valid and relevant by the two experts and the proportion of total items judged content valid. An example of this scale is attached on Appendix A.

\section{Development of Implementation and Evaluation Plan}

The goal for this project is to pilot for a period of 6 months, which would allow area nurse practitioners ample time to integrate it into their diabetic patient exam and screening process. The project will include the assessment and screening tool, algorithm, and treatment and practice guidelines. At the end of the allocated time period, an electronic survey will be dispersed to practitioners via email to provide feedback on the project tools and forms.

\section{Project Dissemination}

Dissemination of the scholarly project is an important step in the DNP project. According to Ahmed, Andrist, Davis \& Fuller, "it is our professional responsibility to share knowledge-knowledge generated from practice" [2]. Among the various methods of disseminating the project, publishing in peer-reviewed journals ranks the most prestigious. This allows the scholar to project findings to professional colleagues rapidly. Another means is through poster presentations and at various conferences as a podium speaker. Regardless of the chosen method of dissemination, the goal is to improve practice and patient safety through the process of developing a scholarly project.
Dissemination of this project included a presentation at the annual nurse practitioner symposium two consecutive years and initially was presented as a project proposal. Throughout the following year, the assessment tool was developed with assistance from my DNP mentor and members of the project team. Final dissemination was conducted via podium presentation, as well as hand out copies of the assessment tool, with question and answer session following. By sharing the project with an area community network of nurse practitioners who are members of a particular region, I contributed to the growth and development of a community organization. This option is frequently overlooked but is an ideal collaboration to improve the overall health and well-being of those patients it serves [5].

\section{Discussion of Findings in Context of Literature}

The assessment and screening tool is an excellent guide to support practitioners in the evaluation of high risk patients such as diabetics suffering from peripheral neuropathy. In today's world of healthcare, providers rely on multiple sources and experts to improve patient care through collaboration [15]. Up until the mid-1990s, Charcot foot was thought of as a rare sensory deficit condition but experts later recognized it as a destructive process, which led to immobilization to prevent further injury or trauma by making the patient non weight-bearing until the acute phase had been resolved [43]. Success of treatment is based on increasing awareness in practitioners who routinely provide care for the diabetic patient population.

One study conducted by Botek, Anderson \& Taylor described a 53 year-old male who was misdiagnosed in the emergency department after presenting with multiple symptoms including pain, redness, and edema to the foot and ankle [8]. This patient was admitted to the hospital and given a course of IV antibiotics, then discharged home with oral antibiotics and instructed to follow up with his primary care physician. After being seen by the PCP 2-3 additional times, the patient was eventually referred to an orthopedic specialist and diagnosed accurately with Charcot foot but the damage suffered to the foot and ankle was irreversible at that point.

It is estimated that 0.1 to $5 \%$ of all diabetics will develop Charcot foot at some point during their disease with an increase in odds for those suffering from end-stage neuropathy. Furthermore, those patients with foot ulcerations are more likely to require extremity amputation; therefore, "it is extremely important for the foot and ankle specialist to judiciously approach the Charcot joint" [7].

As a result of impaired peripheral sensory neuropathy in patients suffering from type 2 diabetes, patients may have no specific recollection of injury. The earliest sign of Charcot foot may include a sudden change in the appearance of the foot or ankle and or discoloration [38]. Therefore, patients often delay seeking medical treatment due to vague symptoms or being unaware they have sustained any type of injury.

The current literature supports the need for further education and assessment tools to aid in the correct diagnosis and treatment 
referrals for patients who are at high risks for developing Charcot foot. It is imperative that practitioners be given every means of identifying these patients and intervening before life threatening complications occur. Currently, there are various advanced assessment tools available but are directed toward the advanced specialist skills.

\section{Project Strengths, Limitations, and Recommendations}

The strengths of the assessment and screening tool include the outlined specific areas of the foot to be assessed and inclusion of any identified abnormalities. Also, a section exists for pertinent patient data to be considered such as latest HgA1C levels, which indicate controlled or uncontrolled blood glucose levels over a three month period. This data is especially important since this directly relates to progression of healing. Additionally, the tool contains instruction for further treatment or referral based on the assessment findings.

Limitations of the project involved an initial negate by one practitioner to accept the terminology of Charcot foot but rather felt it was simply a complication of diabetes and felt it could be treated as such. After further education and the development of the assessment tool, which was presented at the annual nurse practitioner symposium, the project and tool were more widely accepted. Furthermore, various other advanced practice nurses have since voiced an interest in gaining information on how to perform a more structured foot and ankle examination on their diabetic patient population. The interest has been from practitioners within local acute care settings as well as community clinics.

Recommendations for the project were made by members of the area nurse practitioners and consisted of the addition of BMI (body mass index) and specific interpretation of monofilament points to the assessment tool.

\section{Project Summary}

In summary, the DNP project has the potential to impact the diabetic patient population through a process of identifying a need and developing a project to address the issue through evidencebased practice. Charcot foot is a devastating and potential life threatening complication of diabetes and those suffering from peripheral neuropathy. As stated previously, many patients suffering from peripheral neuropathy or impaired sensory perception may experience injuries that are unaware until they begin to see visible signs of trauma such as edema, discoloration, or deformity [13]. For these reasons, patients often times delay seeking treatment or are treated by healthcare providers with little or no knowledge on the clinical manifestations of diabetic foot complications.

If left untreated, Charcot foot may progress to permanent disfigurement or amputations. Early detection and intervention is the key to preventing this serious condition. The development of an assessment and screening tool and following ADA recommendations will assist nurse practitioners and benefit the diabetic population. Currently, there are multiple assessment tools available to specialists in this area such as orthopedics and podiatry but are far more advanced than what is needed for early recognition and referral for advanced practice nurses. After receiving feedback regarding a need from local advanced practice nurses, an assessment and screening tool was developed, along with screening algorithm and assessment and treatment practice guidelines, as a means to assist them in the care of the diabetic patient population who are at risk due to peripheral sensory deficits. In doing so, the goal is for immediate intervention, treatment, and referral to podiatry specialty if warranted and prevent further damage or injury.

\section{References}

1. Aalaa M, Malazy A, T, Sanjari M, Peimani M and Moharjeri-Tehrani M. Nurses' role in diabetic foot prevention and care; a review.Journal of Diabetes and Metabolic Disorders. 2012;11:24. Doi: 10.1186/22516581-11-24

2. Ahmed SW. Capstone Project: Development, Implementation, Evaluation, and Dissemination. In DNP education, practice, and policy: Redesigning advanced practice roles for the 21st century. New York; NY: Springer Pub: 2013.

3. American Association of Colleges of Nursing. The essentials of doctoral education for advanced nursing practice. American Association of Colleges of Nursing. 2006.

4. American Diabetes Association: Living with diabetes; complications. 2013.

5. Anderson BA, Knestrick JM and Barroso R. Implementation and Dissemination of DNP Practice Scholarship. In DNP Capstone Projects: Exemplars of Excellence in Practice. 2014.

6. Barry ME. Better, safer patient care through evidence-based practice and teamwork. The American Nurse. 2014;46(4):12.

7. Bernstein B, Ritter Z, and Diamond, R. Neuropathic Ankle Arthrodesis with Intramedullary Nail Fixation. The Foot and Ankle Online Journal. 2012;5(7):1.

8. Botek G, Anderson MA, and Taylor R. Charcot neuroarthropathy: An often overlooked complication of diabetes. Cleveland Clinic Journal of Medicine. 2010;77(9);593-599. Doi:10.3949/ccjm.77a.09163

9. Chapman Z, Shuttleworth C, and Huber J. High levels of anxiety and depression in diabetic patients with Charcot foot. Journal of Foot and Ankle Research. 2014;7:22. Doi: 10.1186/1757-1146-7-22

10. Charcot Arthropathy.

11. Charcot Foot | Charcot Foot Information | Charcot Foot Treatment | Charcot Foot Prevention | Charcot Foot Symptoms.

12. Charcot-marie-tooth-association: What is charcot-marie-tooth (cmt)?

13. Deformity | Define Deformity at Dictionary.com. (2014).

14. Despins, LA, Scott-Cawiezell J, and Rouder JN. Detection of patient risk by nurses: a theoretical framework. Journal of Advanced Nursing. 2010;66(2):465-474. Doi:10.1111/j.1365-2648.2009.05215.x

15. Evidence-based Practice | Canton Mercy. 2014.

16. Forsyth DM, Wright TL, Scherb CA, and Gaspar PM. Disseminating Evidence-Based Practice Projects: Poster Design and Evaluation. Clin Schol Rev. 2010:3(1):14-21. Doi:10.1891/1939-2095.3.1.14 
17. Fowler M. Diabetes: Magnitude and mechanisms. Clinical Diabetes. 2010;28(1):42-46. Doi:10.2337/diaclin.25.1.25

18. Gouveri E and Papanas N. Charcot osteoarthropathy in diabetes: A brief review with an emphasis on clinical practice. World Journal of Diabetes. 2011;2(5):59-65. Doi:10.4239/wjd.v2.i5.59

19. Houghton VJ, Bower VM, and Chant DC. Is an increase in skin temperature predictive of neuropathic foot ulceration in people with diabetes? A systematic review and meta-analysis. Journal of Foot and Ankle Research J Foot Ankle Res. 6(1), 31. Doi:10.1186/1757-11466-31

20. Jackson K. Charcot Neuroarthropathy. 2011.

21. Kaynak G, Birsel O, Guven MF, and Olgut T. An overview of the Charcot foot pathophysiology. Diabetic Foot and Ankle. 2013;4:10 Doi:10.3402/dfa.v4i0.21117

22. LaVela SL, Gallan AS. Evaluation and Measurement of Patient Experience. Patient Experience Journal. 2014;1(1):1.

23. Lin H and Lorenzo N. Diabetic neuropathy. 2013.

24. Malone R, and Bucknall T. Models and frameworks for implementing evidence-based practice: Linking evidence to action. Malden, MA: Wiley-Blackwell Publishing.

25. Meyers LA. Diabetic Foot: Prevention, Assessment, Treatment and Interventions | Wound Source. 2013.

26. Milne TE, Rogers JR, Kinnear EM, Martin HV, Lazzarini PA, and Boyle FM. Developing an evidence-based clinical pathway for the assessment, diagnosis and management of acute Charcot NeuroArthropathy: a systematic review. Journal of Foot and Ankle Research. 2013;6(1);30. Doi: 10.1186/1757-1146-6-30

27. Mitchell G. Selecting the best theory to implement planned change. Nursing Management. 2013;20(1);32-37. Doi:10.7748/ nm2013.04.20.1.32.e1013

28. Moran KJ, Burson R, and Conrad, D. Driving the Practicum to Impact. In The doctor of nursing practice scholarly project: A framework for success. Burlington; MA: Jones \& Bartlett Learning: 2014.

29. Morgan N. How to do a Semmes Weinstein monofilament exam. Wound Care Advisor. 2013;2(1):23-24.

30. Mumoli N, Camaiti A. Charcot Foot.Canadian Journal of Medicine. 2012;184(12):1392. Doi:10.1503/cmaj.111972
31. Nancarrow SA, Booth A, Ariss S, Smith T, Enderby P, and Roots A. Ten principles of good interdisciplinary team work. Human Resources for Health. 2013;11:19. Doi: 10.1186/1478-4491-11-19

32. Nurse Practitioner Primary Care Competencies in Specialty Areas: Adult, Family, Gerontological, Pediatric, and Women's Health. (2002).

33. O'Rourke I. Model of care for the high risk foot.ANZ Journal of Surgery. 2010;72(4):286.

34. Peng H, Swierzewski S. Charcot foot overview.

35. Polit DF, and Beck CT. The content validity index: Are you sure you know what's being reported? critique and recommendations. Research in Nursing \& Health. 2006;29(5);489-497. Doi:10.1002/ nur.20147

36. Rempher KJ. Putting theory into practice: Six steps to success. American Nurse Today.

37. Rogers L, Frykberg R, Armstrong D, Boulton A, Edmonds M, Ha Van G, et. al. The Charcot foot in diabetes. Diabetes Care. 2011;34(9):21232129. Doi: $10.2337 / \mathrm{dc} 11-0844$

38. Sanders L. Ask the experts: Expert answers about your diabetes from the pages of Diabetes forecast. Alexandria, VA: American Diabetes Association.

39. Sumpio BE. Contemporary Evaluation and Management of the Diabetic Foot. Scientific. 2012(2012);1-17. Doi:10.6064/2012/435487

40. Titler MG, and Moore J. Evidence-Based Practice: A Civilian Perspective. Nursing Research. 2010;59(1): S2-S6. Doi:10.1097/ NNR.0b013e3181c94ec0

41. Type 2 Diabetes.

42. Varaei S, Salsali M, and Heshmat R. Education and implementing evidence-based nursing practice for diabetic patients. Iranian Journal of Nursing and Midwifery Research. 2013;18(3):251-257.

43. Veillette C. Charcot Arthropathy. Orthopaedics OneArticle.

44. Welch, G. W., Jacobson, A. M., \&Weinger, K. The Psychological and Social Impact of Type 2 Diabetes. Health AM. 2008.

45. What is a Podiatrist? | Learn About Feet | APMA. 2014.

46. Zgonis T. How to Manage The Charcot Midfoot Deformity. Podiatry Today. 2010;23(7):68-75 